\title{
Tweeting from the Bench: Twitter and the Physician-Scientist Benefits and Challenges
}

\author{
Jessica S. Little ${ }^{1,2}$ (D) Rizwan Romee ${ }^{2,3}$ \\ Accepted: 4 November 2020 / Published online: 11 November 2020 \\ (C) Springer Science+Business Media, LLC, part of Springer Nature 2020
}

\begin{abstract}
Purpose of Review Social media platforms such as Twitter are increasingly utilized to interact, collaborate, and exchange information within the academic medicine community. However, as Twitter begins to become formally incorporated into professional meetings, educational activities, and even the consideration of academic promotion, it is critical to better understand both the benefits and challenges posed by this platform.

Recent Findings Twitter use is rising amongst healthcare providers nationally and internationally, including in the field of hematology and oncology. Participation on Twitter at national conferences such as the annual meetings of American Society of Hematology (ASH) and American Society of Clinical Oncology (ASCO) has steadily increased over recent years. Tweeting can be used advantageously to cultivate opportunities for networking or collaboration, promote one's research and increase access to other's research, and provide efficient means of learning and educating. However, given the novelty of this platform and little formal training on its use, concerns regarding patient privacy, professionalism, and equity must be considered.

Summary These new technologies present unique opportunities for career development, networking, research advancement, and efficient learning. From "tweet ups" to Twitter journal clubs, physician-scientists are quickly learning how to capitalize on the opportunities that this medium offers. Yet caution must be exercised to ensure that the information exchanged is valid and true, that professionalism is maintained, that patient privacy is protected, and that this platform does not reinforce preexisting structural inequalities.
\end{abstract}

Keywords Twitter $\cdot$ Social media $\cdot$ Technology $\cdot$ Academic medicine

\section{Introduction}

Social media is a rapidly evolving platform for communication that is increasingly being utilized across the academic medicine community. Twitter, a free microblogging platform, enables users to read and post 280-character messages called "Tweets" $[1 \bullet, 2]$. Twitter provides novel opportunities for physician-scientists to interact and collaborate across

This article is part of the Topical Collection on Social Media Impact of Hematologic Malignancies

Jessica S. Little

jlittle@bwh.harvard.edu

1 Division of Infectious Diseases, Brigham and Women's Hospital, 75 Francis Street, PBB A-4, Boston, MA 02115, USA

2 Harvard Medical School, Boston, MA, USA

3 Dana-Farber Cancer Institute, Boston, MA, USA institutions and diverse fields. It increases access to research and enables real-time discussion of new publications [3]. Not only does it serve to disseminate information, it also may be utilized as a means to generate data $[4,5]$. As this platform is increasingly integrated into the academic medical community, it is important to consider both the benefits and potential challenges posed by this technology.

\section{Career Development}

\section{Benefits}

Twitter may promote career development in multiple ways. By providing an open forum that is easily accessible, diverse participants can interface across institutions, academic disciplines, or geographic barriers [6]. In particular, participants may use Twitter as an opportunity to expand their professional network. Colleagues at the same level may use Twitter as an 
opportunity to connect and advance common interests. Even trainees at an early stage are able to follow and engage with leading experts in a particular specialty with greater ease, thus advancing their understanding of key scholarship or topics of discussion at the forefront of the field $[5,6]$.

Additionally, engagement on Twitter prior to and during academic meetings can help build professional relationships and communities that may lead to future collaborations or opportunities for career advancement $[1 \bullet, 2,7]$. In one recent analysis of tweets during the American Society of Clinical Oncology annual meetings between 2011 and 2016, Pemmaraju and colleagues found that both individual authors and overall number of tweets significantly increased over the 5year period [8]. Meeting attendees may tweet responses and commentary to presented scholarship and even arrange "tweet ups" or face-to-face meetings for those who met virtually on Twitter $[5,9]$. And while in the past, missing a national or international conference may have led to loss of access to important new data, ideas, or opportunities for collaboration, now, as academic meetings are increasingly integrated with social media, physicians can watch presentations, participate in discussions, and network with other attendees remotely $[1,10,11]$.

Mentorship and academic sponsorship can also be practiced through the medium of Twitter. Mentors or academic sponsors advanced in their field who have increased influence or impact on Twitter can promote the accomplishments of their mentees to increase their individual visibility. Likewise individuals can promote their own accomplishments including research publications, academic promotions, or awards targeting a broader audience that may result in additional career opportunities $[12,13]$. And as social media engagement continues to grow, academic institutions such as Mayo Clinic have even begun to consider ways to incorporate social media scholarship into metrics for academic promotion and tenure [14•].

\section{Challenges}

While there are considerable potential professional benefits to engaging in social media platforms such as Twitter, there are also challenges. Social media may blur the line between the professional and personal identity of a physician and missteps may harm the professional reputation of users $[15,16]$. It is therefore critical to compose each "Tweet" with the understanding that the post will be public and permanent [5]. In one 2010 study let by Chretian et al., 5156 tweets from selfidentified physicians were analyzed over one month. Of those, 144 tweets were categorized as unprofessional with 38 representing potential patient privacy violations, 33 containing profanity, 14 with sexually explicit material, and 4 with discriminatory statements. And amongst the 27 users responsible for privacy violations, $25(92 \%)$ were identifiable by full listed name on profile, photo, or linked website [17, 18]. Furthermore, physicians are not simply at risk of disapproval by colleagues and patients or punitive actions by employers. A survey of the directors of medical and osteopathic boards revealed $92 \%$ (44 out of 48 respondents) indicating at least one of several online professionalism violations had been reported to the board. In response $71 \%$ held disciplinary hearings and serious disciplinary outcomes including license restriction, suspension, or revocation occurred at $56 \%$ of the boards [18].

In response to these concerns, the American Medical Association created guidelines for social media use amongst physicians [19]. However, this guidance does not provide clear rules of conduct and should serve as simply the first step in the construction of formal policies and training across institutions for physicians on social media.

Another key issue that is introduced by the use of Twitter is the potential amplification of implicit biases and structural inequality already problematic in academic medicine. While many maintain that Twitter can increase equity by opening new channels of communication to diverse individuals across geographic, socioeconomic, and disciplinary barriers, others argue that social media may increase the impact of those who already have the most impact and exacerbate inequality [12]. Gender inequalities have already been identified in many key areas across medicine, and gender bias in the way women are addressed and perceived may affect career advancement [20, 21]. How Twitter reinforces these biases must be considered. One study by Zhu et al. identified Twitter users amongst speakers and coauthors presenting at Academy Health's 2018 Annual Research meeting and evaluated their most recent tweets. Amongst more than 3000 health services researchers, women had less influence on Twitter than men with half of the mean number of followers, and fewer mean likes and retweets per year. These differences were largest amongst full professors and similar across the distribution of number of tweets [22 $]$. Further investigation is needed into whether these inequities exist for other underrepresented minorities on Twitter.

Finally, it is important to acknowledge that Twitter may have detrimental effects on the productivity of participants. While there are small steps being taken towards acknowledging activity and scholarship on social media at certain institutions, there is still minimal formal recognition of physician use of Twitter in a professional sense $[6,14]$. It can be easy to sacrifice the slower more laborious work of designing studies, writing papers or book chapters, and keeping up with patient charting when faced with the potential positive feedback loop of a popular tweet.

\section{Research Advancement}

\section{Benefits}

Social media and Twitter in particular have radically transformed the landscape of information sharing, and this is 
especially relevant in relation to biomedical research. The platform presents opportunities for rapid review of new papers, easy access to multiple journals and expert opinions, increased potential for crowdsourcing, and enhanced postpublication peer review.

Physicians can follow respected journals, professional societies, and mentors or colleagues who may be sharing important advances in the field. In this way, physicians can stay up to date with minimal time expended. Tweets and articles can be saved or "bookmarked" to review in more detail later [5]. Similarly, researchers may increase the impact of their work by using Twitter. One study analyzing 4208 tweets showed that highly tweeted articles were 100 times more likely to be highly cited than less-tweeted articles [23]. Journals may also utilize social media such as Twitter to increase the impact factor of their work. One group recently proposed instituting a TIF or Twitter impact factor for journals to measure the academic reach and impact of a journal on the social media platform [24].

Twitter has also encouraged innovative forms of communicating research findings. Another recent prospective case control crossover study looked at 44 research articles published in the same year in Annals of Surgery. Each article was tweeted in two formats: as the title alone or as the title with a visual abstract. A strong correlation was found between the use of visual abstracts and increased dissemination on social media. Additionally, the articles with a visual abstract tweeted received more site visits than the articles without visual abstracts. [25]

One area that has expanded rapidly on Twitter is postpublication peer review and Twitter journal clubs. Journal clubs have long served as important tools for propagating new research, practicing evidence-based medicine, and developing skills to evaluate research design and validity of the findings [26-28]. Recently a diverse range of Twitter journal clubs have arisen including ID journal club, NephJC, JGIM Twitter Journal Club, and others [27, 28]. Organizers will choose articles and indicate a date and time for the meeting. Tweets are organized and referenced by hashtags and participants can follow along or interact by commenting on individual tweets. Content experts or authors may be invited and physicians at all levels may join in to learn collectively. And while these meetings often cater to physicians and physicianscientists, journal clubs are typically open to any individual including patients, allowing improved public dissemination of new research advances.

Crowdsourcing and collaboration during peer review may lead to important findings of design or methodology errors, statistical inconsistencies, or other flaws in publications. In one case, Twitter critics rapidly identified errors in methodology in an article in Science titled "Genetic Signatures of Exceptional Longevity in Humans". Within a week, the authors released a statement acknowledging a technical error in the lab test used and the paper was eventually retracted [29]. As the speed and breadth of scientific publication increases, Twitter remains an important resource to critically appraise the expanding literature.

Crowdsourcing and network utilization may also be used positively to impact public health efforts by disseminating educational information to communities, amplifying emergency notifications, and enhancing aid efforts when needed [2]. This has been a particularly useful tool during the COVID-19 pandemic of 2020 as the CDC and local health departments have used Twitter to circulate critical health information.

\section{Challenges}

While social media provides an immense opportunity for information uptake and dissemination, there are important caveats to this information exchange. Misinformation is rampant, and developing the ability to discern true facts from misinformation is increasingly challenging as technology advances. New innovations such as the verified badge allow users to know if accounts are authentic, though this may not apply uniformly. As Pershad et al. noted, while a celebrity may be verified due to his/her role in the public eye, that individual's views on healthcare topics such as vaccination may not be valid health information [2].

Additionally, Twitter engagement may be purchased unbeknownst to viewers. In one analysis of the ASCO 2016 annual meeting, the second largest number of retweets was from fake engagement or purchased retweets by a third party [8]. In another study by Desai et al., tweets contained in the official Twitter hashtags of thirteen medical conferences from 2011 to 2013 were analyzed. The Twitter influence of third-party commercial entities was found to be similar to that of healthcare providers [30]. It is critical to curb this fake engagement at professional medical meetings moving forward to reduce bias and promote transparency.

Even if physician accounts and engagement are authentic, financial conflicts of interest are frequently not revealed on social media. This may also lead to bias in transmission of information, particularly if populations with less medical expertise such as patients are involved. In one study in JAMA, 504 out of 634 hematologist-oncologists in the USA who use Twitter were found to have some financial conflicts of interest [31]. However, no clear regulations regarding disclosure exist in regard to physician social media and this should be duly considered when evaluating information sources.

Another example of the potential challenges of Twitter was demonstrated with the rapid increase in preprints over recent years and notably during the COVID-19 pandemic. While preprints are beneficial in making novel findings rapidly available, these manuscripts often have not undergone the full peer review process. Inexperience from the media and lay public in distinguishing peer-reviewed from non-peer-reviewed 
publications can lead to magnification of findings that are erroneous [32, 33].

\section{Efficient Learning}

\section{Benefits}

Twitter not only creates unique opportunities for learning about new research findings, it can also provide rich clinical educational content [34]. "Tweetorials" or threaded tweets are used frequently to present lessons on clinical topics and engage learners at all levels [35]. Teaching podcasts such as "The Curbsiders" and "Clinical Problem Solvers" have also utilized Twitter to widen their audience and condense important lessons into easily digestible tweets.

One systematic review examined 29 studies that assessed the effect of social media platforms on graduate medical education. These modalities were used to share clinical teaching, points, disseminate evidence-based medicine, and circulate conference materials. Given the fast-paced nature of medical residency, social media provides a logical space for on-the-go learning and review. One notable finding was that most studies offered mixed results and provided little guidance on how best to incorporate social media platforms formally into graduate medical education [36].

Not only does Twitter provide opportunities for trainee and continuing medical educations, it also may be used as a critical tool for patient education [37]. In one survey-based study, A Breast Cancer Social Media Twitter support community was created. Respondents reported increased knowledge about their breast cancer in a variety of areas and participation led $31.2 \%$ to seek a second opinion or bring additional information to the attention of their treatment team [38]. On Twitter, communities can be created for and by patients using diseasespecific hashtags [39]. For rare diseases in particular, these communities can facilitate new avenues for connection, education, and collaboration between patients and physicians working in highly specialized areas [40]. These networks can even be used as modes to propagate information about available clinical trials to diverse populations [41].

\section{Challenges}

Important limitations to learning via Twitter remain. Patient privacy issues can arise, particularly as photos, radiology, and case descriptions are more widely shared [2, 5, 42]. Twitter can serve as an echo chamber, where ideas are magnified by like-minded individuals in close networks, reducing the sharing of outside perspectives [6]. Finally, the volume of information can overwhelm users, making it difficult to distinguish valuable knowledge from irrelevant comments.

\section{Conclusion}

There are significant benefits to the effective utilization of social media platforms such as Twitter. Physicians and scientists may grow their networks, gain career opportunities, expand the impact of their research, connect with patients, stay up to date on novel discoveries, and much more. However, clear frameworks for professional use of this technology are still being developed. It is vital to better understand the risks to patients and providers in order to safely and deliberately integrate this valuable tool into our institutions and practices.

\section{Compliance with Ethical Standards}

Conflict of Interest The authors declare that there is no conflict of interest.

Human and Animal Rights and Informed Consent This article does not contain any studies with human or animal subjects performed by any of the authors.

\section{References}

Papers of particular interest, published recently, have been highlighted as:

- Of importance

•- Of major importance

1. Pemmaraju N, Mesa RA, Majhail NS, Thompson MA. The use and impact of Twitter at medical conferences: Best practices and Twitter etiquette. Semin Hematol. 2017;54:184-8 Showed major increase in number of tweets at the American Society of Clinical Oncology annual meetings between 2011 and 2016.

2. Pershad Y, Hangge PT, Albadawi H, Oklu R. Social medicine: Twitter in healthcare. J Clin Med. 2018;7. https://doi.org/10.3390/ jcm7060121.

3. Savage N. Scientists in the Twitterverse. Cell. 2015;162:233-4.

4. Thompson MA, Ahlstrom J, Dizon DS, Gad Y, Matthews G, Luks HJ, et al. Twitter 101 and beyond: introduction to social media platforms available to practicing hematologist/oncologists. Semin Hematol. 2017;54:177-83.

5. Attai DJ, Anderson PF, Fisch MJ, Graham DL, Katz MS, Kesselheim J, et al. Risks and benefits of twitter use by hematologists/oncologists in the era of digital medicine. Semin Hematol. 2017;54:198-204.

6. Choo EK, Ranney ML, Chan TM, Trueger NS, Walsh AE, Tegtmeyer K, et al. Twitter as a tool for communication and knowledge exchange in academic medicine: a guide for skeptics and novices. Med Teach. 2015;37:411-6.

7. Chaudhry A, Glodé LM, Gillman M, Miller RS. Trends in twitter use by physicians at the American society of clinical oncology annual meeting, 2010 and 2011. J Oncol Pract. 2012;8:173-8.

8. Pemmaraju N, Thompson MA, Mesa RA, Desai T. Analysis of the use and impact of Twitter during American Society of Clinical Oncology annual meetings from 2011 to 2016: focus on advanced metrics and user trends. J Oncol Pract. 2017;13:e623-31.

9. Thompson MA, Majhail NS, Wood WA, Perales M-A, Chaboissier M. Social media and the practicing hematologist: Twitter 101 for 
the busy healthcare provider. Curr Hematol Malig Rep. 2015;10: 405-12.

10. Tanoue MT, Chatterjee D, Nguyen HL, Sekimura T, West BH, Elashoff D, et al. Tweeting the meeting. Circ Cardiovasc Qual Outcomes. 2018;11:e005018.

11. Brown SA, Daly RP, Duma N, Yang EH, Pemmaraju N, Parwani P, et al. Leveraging social media for cardio-oncology. Curr Treat Options in Oncol. 2020;21. https://doi.org/10.1007/s11864-02000775-3.

12. Klar S, Krupnikov Y, Ryan JB, Searles K, Shmargad Y. Using social media to promote academic research: identifying the benefits of twitter for sharing academic work. PLoS One. 2020;15: e0229446.

13. Jordan K, Weller M. Academics and social networking sites: benefits, Problems and Tensions in Professional Engagement with Online Networking. J Interact Media Educ. 2018;2018. https:// doi.org/10.5334/jime.448.

14. Cabrera D, Vartabedian BS, Spinner RJ, Jordan BL, Aase LA, Timimi FK. More Than Likes and Tweets: Creating Social Media Portfolios for Academic Promotion and Tenure. J Grad Med Educ. 2017;9:421-5 First demonstration of one academic institution's consideration of incorporation of social media scholarship into academic promotion.

15. Mostaghimi A, Crotty BH. Professionalism in the digital age. Ann Intern Med. 2011;154:560-2.

16. DeCamp M, Koenig TW, Chisolm MS. Social media and physicians' online identity crisis. JAMA. 2013;310:581-2.

17. Chretien KC, Azar J, Kind T. Physicians on Twitter. JAMA - J Am Med Assoc. 2011;305:566.

18. Greysen SR, Chretien KC, Kind T, Young A, Gross CP. Physician violations of online professionalism and disciplinary actions: a national survey of state medical boards. JAMA. 2012;307:1141-2.

19. Shore R, Halsey J, Shah K, Crigger B-J, Douglas SP. Report of the AMA council on ethical and judicial affairs: professionalism in the use of social media. J Clin Ethics. 2011;22:165.

20. Duma N, Durani U, Woods CB, et al. Evaluating unconscious bias: speaker introductions at an international oncology conference. In: J. Clin. Oncol. American Society of Clinical Oncology; 2019. p. 3538-45.

21. Duma N. Gender differences in publication rates in oncology: looking at the past, present, and future. Cancer. 2020;126:2759-61.

22. Zhu JM, Pelullo AP, Hassan S, Siderowf L, Merchant RM, Werner RM. Gender differences in Twitter use and influence among health policy and health services researchers. JAMA Intern Med. 2019;179:1726 First scientific analysis of potential gender bias amongst physicians who use Twitter.

23. Eysenbach G. Can tweets predict citations? Metrics of social impact based on Twitter and correlation with traditional metrics of scientific impact. J Med Internet Res. 2011;13:e123.

24. Cardona-Grau D, Sorokin I, Leinwand G, Welliver C. Introducing the Twitter impact factor: an objective measure of urology's academic impact on Twitter. Eur Urol Focus. 2016;2:412-7.

25. Ibrahim AM, Lillemoe KD, Klingensmith ME, Dimick JB. Visual abstracts to disseminate research on social media. Ann Surg. 2017;266:e46-8.
26. Linzer M. The journal club and medical education: over one hundred years of unrecorded history. Postgrad Med J. 1987;63:475-8.

27. Topf JM, Sparks MA, Phelan PJ, Shah N, Lerma EV, GrahamBrown MPM, et al. The evolution of the journal club: from Osler to Twitter. Am J Kidney Dis. 2017;69:827-36.

28. Mehta N, Flickinger T. The times they are a-changin': academia, social media and the JGIM Twitter journal Club. J Gen Intern Med. 2014;29:1317-8.

29. Mandavilli A. Peer review: trial by Twitter. Nature. 2011;469:2867.

30. Desai T, Dhingra V, Shariff A, Shariff A, Lerma E, Singla P, et al. Quantifying the Twitter influence of third party commercial entities versus healthcare providers in thirteen medical conferences from 2011-2013. PLoS One. 2016;11:e0162376.

31. Tao DL, Boothby A, McLouth J, Prasad V. Financial conflicts of interest among hematologist-oncologists on Twitter. JAMA Intern Med. 2017; 177:425.

32. Callaway E. Will the pandemic permanently alter scientific publishing? Nature. 2020;582:167-8.

33. Kwon D. How swamped preprint servers are blocking bad coronavirus research. Nature. 2020;581:130-1.

34. Thamman R, Gulati M, Narang A, Utengen A, Mamas MA, Bhatt DL. Twitter-based learning for continuing medical education? Eur Heart J. 2020. https://doi.org/10.1093/eurheartj/ehaa346.

35. Breu AC. From Tweetstorm to Tweetorials: threaded tweets as a tool for medical education and knowledge dissemination. Semin Nephrol. 2020;40:273-8.

36. Sterling M, Leung P, Wright D, Bishop TF. The use of social media in graduate medical education. Acad Med. 2017;92:1043-56.

37. Sugawara Y, Narimatsu H, Hozawa A, Shao L, Otani K, Fukao A. Cancer patients on Twitter: a novel patient community on social media. BMC Res Notes. 2012;5:699.

38. Attai DJ, Cowher MS, Al-Hamadani M, Schoger JM, Staley AC, Landercasper J. Twitter social media is an effective tool for breast cancer patient education and support: patient-reported outcomes by survey. J Med Internet Res. 2015;17:e188.

39. Katz MS, Utengen A, Anderson PF, Thompson MA, Attai DJ, Johnston C, et al. Disease-specific hashtags for online communication about cancer care. JAMA Oncol. 2016;2:392.

40. Pemmaraju N, Utengen A, Gupta V, Kiladjian J-J, Mesa R, Thompson MA. Rare cancers and social media: analysis of Twitter metrics in the first 2 years of a rare-disease community for myeloproliferative neoplasms on social media- \#MPNSM. Curr Hematol Malig Rep. 2017;12:598-604.

41. Sedrak MS, Cohen RB, Merchant RM, Schapira MM. Cancer communication in the social media age. JAMA Oncol. 2016;2:822.

42. Crane GM, Gardner JM. Pathology image-sharing on social media: recommendations for protecting privacy while motivating education. AMA J Ethics. 2016;18:817-25.

Publisher's Note Springer Nature remains neutral with regard to jurisdictional claims in published maps and institutional affiliations. 